\title{
Die EU als Dauerreformprojekt
}

\section{Politische Ordnungsmodelle und ihre Implikationen}

\section{Warum Dauerreform?}

Im Zuge der nun schon mehrere Jahre andauernden Eurokrise wird die Kritik an der EU zahlreicher und lauter. Das ist vor allem für Deutschland eine neue Erfahrung, weil hier länger als in den anderen großen Mitgliedstaaten die EU sowohl bei den politischen Parteien als auch in der Bevölkerung als alternativlos galt und als ein Projekt, das weiter gestärkt werden müsse. In Frankreich dagegen war und ist die EU seit Langem heiß umstritten. De Gaulles „Politik des leeren Stuhls“ - der fast einjährige Boykott der EU-Gremien durch Frankreich - wollte eine völlig andere EU erreichen als in den Verträgen vorgesehen. Sie war nicht die erste Initiative ihrer Art und stürzte die EU Mitte der 1960er in eine massive Krise. Gut 10 Jahre früher war bereits die ursprünglich von der französischen Regierung vorgeschlagene Europäische Verteidigungsgemeinschaft in der französischen Nationalversammlung in einer dramatischen Sitzung gescheitert, nachdem sie von den übrigen Gründungsmitgliedern bereits ratifiziert worden war.

In Großbritannien spaltet die EU nicht erst seit David Cameron politische Parteien und Bevölkerung. Nachdem die konservative Regierung Heath nach zwei vergeblichen Versuchen, die von de Gaulle blockiert wurden, schließlich den Beitritt erreicht hatte, verlor sie die Unterhauswahlen. Die neugewählte Labor-Regierung unter Wilson war über die EU-Mitgliedschaft in Parteiführung, Parlamentsfraktion und Mitgliedschaft so komplett zerstritten, dass sie sich nur mit einem Referendum $\mathrm{zu}$ helfen wusste, das im Mutterland der Parlamentssouveränität eigentlich ein Fremdkörper ist. Später spaltete sich eine pro-europäische sozialdemokratische Partei von der zunehmend europakritischen Laborpartei ab, während heute die Konservativen durch europakritische Strömungen innerhalb und außerhalb der Partei unter Druck sind. Massive Krisen und heftige Grundsatzdebatten sind in der EU also nicht neu, sondern der Regelfall.

Parallel dazu ergießt sich eine Flut von Reformvorschlägen über das Publikum, und zwar seit über 50 Jahren. Einige sind praktischerweise in dicken Sammelbänden abgedruckt (Lipgens 1986). Im Internetzeitalter macht sich niemand mehr diese Mühe, aber die Zahl der Vorschläge ist gefühlt seitdem nicht geringer geworden. 
Reformiert werden soll alles und jedes, von der Grundstruktur der EU (Staatenbund oder Bundesstaat oder etwas ganz anderes?) über einzelne Politikfelder (die gemeinsame Außen- und Sicherheitspolitik ist neben der Agrarpolitik ein Dauerbrenner) zur demokratischen Kontrolle (durch Europaparlament, nationale Parlamente, Zivilgesellschaft, Direktwahl des Kommissionspräsidenten?). Die immer länger werdenden Verträge sollen konsolidiert und vereinfacht werden (und werden doch immer länger), die Finanzverfassung reformiert und die innere Sicherheit verbessert, alles unter Wahrung der Eigenstaatlichkeit und kulturellen Identität der Mitgliedstaaten und natürlich ohne weitere Kosten und Personal. Seit vielen Jahrzehnten immer wieder spannend ist die Diskussion darüber, wo das Europaparlament seinen Sitz haben und wer darüber befinden sollte. Man stelle sich vor, Deutschland wäre dem gleichen Reform-Furor ausgesetzt!

Es geht aber nicht nur um Worte, sondern auch um Taten. Seit Mitte der 1980er Jahre finden alle paar Jahre große Regierungskonferenzen statt, die die Verfassungsstruktur der EU verändern. Und was sich nicht durch große Regierungskonferenzen oder kleine Vertragsrevisionen ändern lässt, wird durch Richterrecht (zusammenfassend Alter 2009) oder durch inkrementelle Anpassungsprozesse am Rande alltäglicher Politik (Héritier 2007) reformiert. Selbst vor staatlichen Kernaufgaben macht die EU nicht halt (Genschel/Jachtenfuchs 2014).

Aber woher kommt dieser Trend zur Dauerreform? Diese Frage wird natürlich in der Integrationstheorie seit Jahrzehnten diskutiert und wird auch hier nicht endgültig beantwortet werden. Für die gegenwärtige Debatte scheinen mir jedoch drei Faktoren besonders wichtig, die mögliche Reformkorridore bestimmen, vor allem in den Bereichen, die durch die Eurokrise besonders betroffen sind:

- die existierende institutionelle Struktur, die teilweise immer noch durch die Politikverflechtungsfalle gekennzeichnet ist;

- die Notwendigkeit zur Bearbeitung der Folgeprobleme früherer Integrationsschritte (Spill-over);

- zunehmend europaskeptische Wähler (constraining dissensus).

Die Politikverflechtungsfalle wird im Normalbetrieb der EU zwar durch das Vorschlagsmonopol der Kommission und durch supranationales Richterrecht des EuGH konterkariert (Scharpf 2006). Nach wie vor sind aber die gliedstaatlichen Regierungen im Ministerrat und im Europäischen Rat Teil des gemeinschaftlichen Entscheidungssystems und vertreten dort ihre institutionellen Eigeninteressen. Zwar bewegt sich der Entscheidungsprozess langsam von der Einstimmigkeit weg, aber er ist formal wie praktisch noch weit von einfachen Mehrheitsentscheidungen entfernt - und durch die Stärkung des Europäischen Rates in der Krise steigt die Bedeutung der Quasi-Einstimmigkeit wieder. 
Folgeprobleme früherer Integrationsschritte erhöhen aber den Druck, neue Regeln und Institutionen zu schaffen, die die Folgen der gewachsenen Interdependenz einhegen. Diese gestiegene Nachfrage nach grenzüberschreitenden Regeln bei gesteigerter Interdependenz ist die Kernaussage sowohl der neofunktionalistischen Integrationstheorie (Haas 2004) wie auch der Regimetheorie in den internationalen Beziehungen (Keohane 1984). Der Knackpunkt in der EU besteht darin, dass die Steigerung der Interdependenz in der EU nicht exogen, sondern endogen ist - sie ist ein seit Jahrzehnten bewusst vorangetriebenes politischen Projekt, mit dem die Schutzfunktion staatlicher Grenzen immer weiter reduziert wird im Gegenzug für Wohlfahrtsgewinne und weltpolitische Gestaltungsfähigkeit. Der entscheidende Faktor in der gegenwärtigen EU besteht darin, dass integrationspolitisches Handeln vor dem Hintergrund einer jahrzehntelangen Integrationsgeschichte stattfindet, die ein großes und wirkungsmächtiges Institutionen- und Regelsystem geschaffen hat. Nichtstun ist deshalb keine Option, weil sie nicht staatliche Autonomie schützt, sondern einen schon hochgradig europäisch bestimmten Status quo. Das kann man sehr schön an der Steuerpolitik sehen: Eigentlich wollen alle Mitgliedstaaten nur eine sachlich eng begrenzte Minimalharmonisierung. Tatsächlich aber ist die europäische Steuerpolitik hochgradig ausdifferenziert und invasiv, weil die Folgeprobleme früherer EU-Entscheidungen selbst wieder nur durch neue EU-Entscheidungen angegangen werden könnten (Genschel/Jachtenfuchs 2011).

Das ist die Logik des altbekannten Elitenmodells, das in kleinen Schritten zu immer mehr Integration führt. Seit längerer Zeit zerbröselt aber der frühere ,permissive Konsens“ und wird durch den einengenden Dissens ersetzt (Hooghe/Marks 2009). Durch Referenden, Wahlen und die Restrukturierung der Parteiensysteme wird der Entscheidungsprozess komplexer und zwingt die EU-Organe inklusive des Rates, die Reaktionen des Publikums mit zu antizipieren. Das hat spezifische Folgen für den Verlauf von Reformdiskussionen.

\section{Welche grundlegenden Optionen gibt es?}

Hier geht es darum, sich nicht in den Details zahlloser Vorschläge zu verlieren, sondern einen Schritt zurückzutreten und grundlegende Optionen der EU in den Blick zu nehmen.

Die erste ist der Weg zurück - nicht zum Europa souveräner Nationalstaaten, das faktisch passé ist, sondern zur Wirtschaftsverfassung (Mestmäcker 1984) und zum klassischen Regulierungsmodell (Majone 1996). Das ist für viele das Modell der Gründungsverträge. Danach soll sich die EU auf die Setzung und Durchsetzung von Regeln für den Binnenmarkt konzentrieren. Ihr Mandat ist in dieser Sicht die Er- 
richtung einer liberalen Rahmenordnung für den Markt, die sich durchaus nicht auf Wettbewerbsregeln beschränken muss, sondern auch marktrelevante flankierende Politiken wie Umweltpolitik, Sozialstandards, Verbraucherschutz etc. mit berücksichtigen kann und soll. Die Vertreter dieser Sichtweise berufen sich dabei auf das Mandat der Gründungsverträge und auf die neuere Diskussion über die Grenzen demokratischer Verantwortung in der EU, wonach diese nicht belastbar genug ist, um massive wirtschaftliche Umverteilung, Militäreinsätze oder tiefe Eingriffe in die persönliche Freiheit zu legitimieren. Die Eurokrise zeigt aus dieser Perspektive, dass die EU ihr Mandat überschritten hat, und die Handlungsempfehlung lautet, zu den bewährten Wurzeln zurückzukehren.

Die zweite ist der Weg nach vorne, hin zu einem föderalen Europa. Um den Föderalismus als Orientierungsmodell für die EU ist es in den letzten Jahren ruhig geworden. $\mathrm{Zu}$ unrealistisch erschien die in den Anfängen der Integration vielfach erhobene Forderung nach einem europäischen Bundesstaat. Selbst die CDU, die als Europapartei par excellence jahrzehntelang die Forderung nach einem europäischen Bundesstaat mit Polizeigewalt, Armee, Steuern und Parlament in ihren Programmen hatte, hat davon schrittweise Abstand genommen (Jachtenfuchs 2002). Angesichts zunehmender Europaskepsis in der Bevölkerung, der vehementen Ablehnung des „F-words“ durch einige Mitgliedstaaten und der Skepsis des Bundesverfassungsgerichts scheint die große föderalistische Lösung völlig außerhalb der politischen Reichweite zu sein. Allerdings hat der politische Föderalismus immer argumentiert, dass die technokratische EU à la Wirtschaftsverfassung oder Regulierungsstaat institutionell die schlechteste aller denkbaren Lösungen sei, da sie starke Institutionen mit schwacher demokratischer Kontrolle vorsehe und zudem ein instabiler Zwischenzustand sei. Die konkreten föderalistischen Vorschläge sehen deshalb nicht mehr die unqualifizierte Staatswerdung der EU vor, sondern beschränken sich auf punktuelle Weiterentwicklungen. Diese sind einerseits die Stärkung der demokratischen Komponente der EU durch die politische Kontrolle der Exekutive (Kommission) und andererseits die Schaffung größerer und leichter verfügbarer Finanzressourcen der EU. Der Eurokrise kann in dieser Perspektive nur durch eine gleichzeitige Stärkung von Demokratie und Handlungsfähigkeit begegnet werden.

Die dritte Option ist das „weiter so“. Während sich die ersten beiden Optionen auf alte Denkfiguren in der Integrationsdebatte zurückführen lassen, ist das hier scheinbar nicht der Fall. Diese Option gibt sich pragmatisch und scheint sich nicht zwischen starken alternativen Ordnungsmodellen zu entscheiden, sondern die endgültige Gestalt der EU weiter offenhalten zu wollen. Das klingt ein bisschen nach Helmut Schmidts Diktum, dass, wer Visionen hat, zum Arzt gehen solle. Dahinter steht jedoch ein profiliertes verfassungspolitisches Modell (Genschel/Jachtenfuchs 
2014), das Merkmale der ersten beiden Optionen auf spezifische Weise kombiniert. Vom föderalen Modell übernimmt es das Einwirken der EU auch auf staatliche Kernbereiche wie Militär, Finanzen und Verwaltung (vgl. für den amerikanischen Fall Skowronek 1982), aber auch Steuern und innere Sicherheit. Vom Modell der Wirtschaftsverfassung übernimmt es die regulative Form der Einwirkung, die mit qualifizierter Mehrheit beschlossen und durch den EuGH kontrolliert wird. Im Gegensatz zum föderalen Modell werden keine großen neuen und gut sichtbaren Institutionen und Handlungskapazitäten geschaffen. Eine europäische Armee oder ein europäisches FBI mit Exekutivbefugnissen stehen nicht auf der Tagesordnung, ebenso wenig signifikante europäische Steuern oder ein europäischer Wohlfahrtsstaat. All dies verbleibt den Staaten, die durch die Stärkung des Europäischen Rates scheinbar die Oberaufsicht führen (Puetter 2012). Im Gegensatz zum Modell der Wirtschaftsverfassung bleiben die Handlungen der EU nicht auf den Bereich der Markregulierung beschränkt, sondern beziehen auch Kernbereiche von Staatlichkeit ein, in der gleichen regulativen Form wie bei der Marktgestaltung. Die EU sieht dann nicht viel anders aus als früher. Sie erwirbt nicht die Symbole von Staatlichkeit (Flagge, Hymmne, Minister oder Gesetze) und nicht die autonome Verfügung über große Gewalt- und Geldmittel. Aber sie reguliert nun auch zunehmende Teile des nationalen Gewaltmonopols (Herschinger/Jachtenfuchs/Kraft-Kasack 2011) und der nationalen Haushalte, Letzteres in einem Ausmaß, das im Bundesstaat USA undenkbar wäre (Hallerberg 2014).

\section{Welche Chancen und Folgen haben diese Optionen?}

Das Modell der Wirtschaftsordnung ist intellektuell anspruchsvoll und zumindest auf den ersten Blick normativ plausibel, denn es bietet eine der wenigen Möglichkeiten, eine politische Ordnung jenseits des Staatsmodells zu denken, das weder die EU auf einen Staatenbund reduziert noch Staatlichkeit auf europäischer Ebene rekonstituieren will, sondern eine starke europäische Marktregulierung mit starker europäischer Staatlichkeit verbindet. Auf den zweiten Blick ist es aber ein technokratisches Modell, das zentrale politische Entscheidungen als unpolitisch umzudefinieren versucht (Joerges 2012). Vor allem ist es von der Wirklichkeit längst überholt: Die EU stellt nicht nur eine Ordnung für Marktteilnehmer dar, sondern reguliert auch Kernbereiche von Staatlichkeit. Die Staaten erheben zwar weiter als einzige Steuern und trotz vielfacher Forderungen sieht es nicht so aus, als würde sich die EU diese Finanzierungsquelle auf absehbare Zeit erschließen können. Aber die EU reguliert Bemessungsgrundlage, Steuerarten und Steuersätze der Mitgliedstaaten bis ins Detail. Dass die Mitgliedstaaten in der Steuerpolitik nur einen begrenzten 
Spielraum haben liegt nicht nur am Druck der Märkte, sondern am dichten Geflecht europäischer Regelungen und Rechtsprechung.

Die EU hat keine eigene Polizeibehörde mit Verhaftungsbefugnissen wie das FBI. Aber mit dem europäischen Haftbefehl und weiteren nach seinem Modell geformten Regelungen braucht sie diese auch nicht unbedingt, sondern überträgt das Prinzip der gegenseitigen Anerkennung vom Bereich des Binnenmarktes auf das Strafrecht. Ihr Haushalt ist immer noch auf ein Prozent ihrer Wirtschaftsleistung beschränkt und damit weit entfernt von den Budgets klassischer Bundesstaaten, die ein Vielfaches dessen betragen. Aber mit den im Zuge der Eurokrise erlassenen Maßnahmen (Sixpack, Fiskalpakt etc.) reguliert sie immer stärker die staatliche Ausgabenpolitik.

Die höchste Wahrscheinlichkeit hat deshalb das dritte Modell, der umfassende Regulierungsstaat. Es wird durch die anfänglich genannten drei Bedingungen gefördert (vgl. ausführlich Genschel/Jachtenfuchs 2013). Durch die Politikverflechtungsfalle gibt es keine Mehrheit für den institutionellen „Großen Sprung“, die föderale Option, da diese den institutionellen Eigeninteressen der Regierungen diametral entgegengesetzt ist. Stattdessen dominieren konfliktminimierende Entscheidungsmuster. Regulierung hat auch deshalb den Vorrang vor dem Aufbau eigener Handlungskapazitäten auf EU-Ebene, weil sie weniger sichtbar ist. Dem Bürger tritt nicht eine europäische Staatsmacht gegenüber, die verhaftet, zum Wehrdienst einzieht, besteuert oder umverteilt, sondern nach wie vor der eigene, in demokratischen Wahlen kontrollierte Staat. So lassen sich die (selbstgemachten) Probleme hoher Interdependenz in der EU und vor allem in der Eurozone mit konfliktminimierenden Mechanismen angehen, ohne die skeptischen Wähler allzu heftig zu provozieren.

Durch die hohe Sichtbarkeit des Europäischen Rates wird der Anschein einer zunehmenden Intergouvernementalisierung, einer Abkehr von der Gemeinschaftsmethode und der Dominanz der (großen) Staaten erzeugt. Aber der Europäische Rat muss weitestgehend im Konsens entscheiden und hat keinen Verwaltungsunterbau. Die klassischen Werkzeuge der Integrationspolitik werden deshalb weiter genutzt: Konfliktminimierung durch Regulierung und durch unvollständige Verträge, deren Überwachung und Durchsetzung der Kommission (Haushaltsüberwachung!) und dem EuGH überlassen wird. Hinzu kommt der gewaltige Bedeutungs- und Aufgabenzuwachs der supranationalen EZB.

Die Entwicklung der EU verläuft dabei anders als die klassischer „,coming-together federations" (Stepan 1999). Die USA oder die Schweiz waren wenige Jahrzehnte nach ihrer Gründung mitnichten die entwickelten Bundesstaaten von heute. Es gelang ihnen aber schließlich, die Verfügung über Gewalt- und Steuermittel zu erringen und einen substanziellen autonomen Verwaltungsapparat aufzubauen. In der EU sind alle drei Ressourcen aufgrund ihrer Entscheidungsstruktur und aufgrund 
des „einengenden Dissenses“ blockiert, wobei aufgrund des Druckes durch die Eurokrise behutsame Entwicklungen im fiskalischen Bereich noch am wahrscheinlichsten sind und mit dem ESM bereits begonnen haben.

Das Ergebnis ist ein Modell, das die Nachteile der Wirtschaftsverfassung und der föderalen Option kombiniert: Die EU bleibt technokratisch und exekutivlastig (Curtin/Egeberg 2009), dringt aber gleichzeitig in staatliche Kernbereiche vor (Genschel/Jachtenfuchs 2014). Es kommt den institutionellen Eigeninteressen der Regierungen und den skeptischen Wählern entgegen, hält die zugrunde liegende Problematik aber offen. Das gibt es sonst nirgendwo. Insofern ist das wahrscheinlichste Ergebnis der europäischen Dauerreform innovativ. Befriedigend ist es nicht.

\section{Literatur}

Alter, Karen J., 2009: The European Court's Political Power. Selected Essays, Oxford.

Curtin, Deirdre/Egeberg, Morten (Hrsg.), 2009: Towards a New Executive Order in Europe?, London.

Genschel, Philipp/Jachtenfuchs, Markus, 2011: How The European Union Constrains the State. Multilevel Governance of Taxation, in: European Journal of Political Research 50 (3), 293-314.

Genschel, Philipp/Jachtenfuchs, Markus, 2013: Alles ganz normal! Eine institutionelle Analyse der Euro-Krise in: Zeitschrift für Internationale Beziehungen 20 (1), 75-88.

Genschel, Philipp/Jachtenfuchs, Markus (Hrsg.), 2014: Beyond the Regulatory Polity? The European Integration of Core State Powers, Oxford.

Haas, Ernst B., 2004: The Uniting of Europe. Political, Economic, and Social Forces 1950-1957, Notre Dame (3. Aufl.; zuerst 1958).

Hallerberg, Mark, 2014: Why is there Fiscal Capacity but little Regulation in the US, but Regulation and little Fiscal Capacity in Europe? The Global Financial Crisis as a Test Case, in: Philipp Genschel/Markus Jachtenfuchs (Hrsg.), Beyond the Regulatory Polity? The European Integration of Core State Powers, 87-104. Héritier, Adrienne, 2007: Explaining Institutional Change in Europe, Oxford.

Herschinger, Eva/Jachtenfuchs, Markus/Kraft-Kasack, Christiane, 2011: Scratching the Heart of the Artichoke? How International Institutions and the EU Constrain the State Monopoly of Force, in: European Political Science Review 3 (3), 445-468. 
Hooghe, Liesbet/Marks, Gary, 2009: A Postfunctionalist Theory of European Integration. From Permissive Consensus to Constraining Dissensus, in: British Journal of Political Science 39 (1), 1-23.

Jachtenfuchs, Markus, 2002: Die Konstruktion Europas. Verfassungsideen und institutionelle Entwicklung, Baden-Baden.

Joerges, Christian, 2012: Europas Wirtschaftsverfassung in der Krise, in: Der Staat 51 (3), 357-385.

Keohane, Robert O., 1984: After Hegemony. Cooperation and Discord in World Politics, Princeton.

Lipgens, Walter (Hrsg.), 1986: 45 Jahre Ringen um die Europäische Verfassung. Dokumente 1939-1984. Von den Schriften der Widerstandsbewegung bis zum Vertragsentwurf des Europäischen Parlaments, Bonn.

Majone, Giandomenico, 1996: The European Community as a Regulatory State, in: Collected Courses of the Academy of European Law 5, 321-419.

Mestmäcker, Ernst-Joachim, 1984: Recht und ökonomisches Gesetz. Über die Grenzen von Staat, Gesellschaft und Privatautonomie, Baden-Baden.

Puetter, Uwe, 2012: Europe's Deliberative Intergovernmentalism. The Role of the Council and European Council in EU Economic Governance, in: Journal of European Public Policy 19 (2), 161-178.

Scharpf, Fritz W., 2006: The Joint-decision Trap Revisited, in: Journal of Common Market Studies 44 (4), 845-864.

Skowronek, Stephen, 1982: Building a New American State. The Expansion of National Administrative Capacities 1877-1920, Cambridge.

Stepan, Alfred, 1999: Federalism and Democracy. Beyond the U.S. Model, in: Journal of Democracy 10 (4), 19-34.

Korrespondenzanschrift:

Prof. Dr. Markus Jachtenfuchs

Professor of European \& Global Governance

Hertie School of Governance

Friedrichstraße 180

10117 Berlin

E-Mail: jachtenfuchs@hertie-school.org 